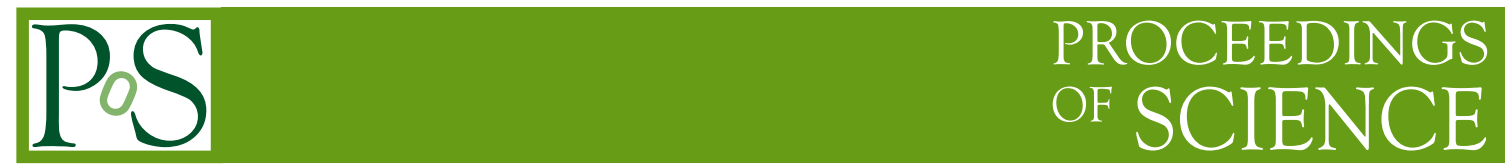

\title{
Strange baryonic resonances and kaonic bound states
}

\author{
Kirill Lapidus for the HADES Collaboration \\ TU München \\ Excellence Cluster "Universe" \\ Boltzmannstr. 2 \\ D-85748 Garching \\ E-mail: kirill.lapiduseph.tum.de
}

Recent results of the HADES collaboration on the low-mass $\Lambda(1405)$ observed in proton-proton collisions at $3.5 \mathrm{GeV}$ in the charged decay mode are discussed together with the status of the searches for the kaonic bound state $p p K^{-}$in the reaction $p p \rightarrow p K^{+} \Lambda$.

XV International Conference on Hadron Spectroscopy-Hadron 2013

4-8 November 2013

Nara, Japan 


\section{Introduction}

The low-energy antikaon-nucleon interaction is strongly affected by the presence of the strange baryonic resonances $\Sigma(1385)$ and $\Lambda(1405)$. The latter is of particular importance, since it is located just below the $\bar{K} N$ threshold. In modern theoretical analyses, the $\Lambda(1405)$ emerges as a dynamically generated state that couples to two poles $[1,2]$. The first narrow pole is mainly associated with the $\bar{K} N$ bound state and located at energies of around $1420 \mathrm{MeV}$, whereas the second broad pole appears as a $\Sigma \pi$ resonance situated at lower energies $(1390 \mathrm{MeV})$. A renewed interest in the $\Lambda(1405)$ rose due to a hypothesis that it might act as a doorway for the formation of a 3-particle cluster that is formed by two nucleons bound by an antikaon - a so-called $p p K^{-}$state $[3,4,5]$.

In this context, the HADES collaboration addressed the low-energy $\bar{K} \mathrm{~N}$ interaction in the following analyses of data collected in proton-proton collisions at beam energy of $3.5 \mathrm{GeV}: 1$ ) analysis of the $\Sigma(1385)^{+}$as a benchmark measurement [6],2) extraction of the $\Sigma(1385)^{0}$ contribution as an important background channel for the $\Lambda(1405)$ analysis [7], 3) reconstruction of the $\Lambda(1405)$ line shape in the charged decay mode $\left.\Lambda(1405) \rightarrow \Sigma^{ \pm} \pi^{\mp}[8,9], 4\right)$ search for the antikaonic-bound state $p p K^{-}$with the help of a Partial Wave Analysis. In this contribution we concentrate on the two last items.

\section{The HADES detector}

Data for the analysis were collected with the High-Acceptance Di-Electron Spectrometer (HADES). This is a versatile charged-particle detector currently operating at the SIS18 synchrotron (GSI Helmholtzzentrum, Darmstadt) in the region of beam kinetic energies of 1-2 GeV/nucleon for nucleus-nucleus collisions, up to $3.5 \mathrm{GeV}$ in proton-induced reactions. The detector covers polar angles from $18^{\circ}$ to $85^{\circ}$ degrees and a large portion of the azimuthal angle; the momentum resolution of the spectrometer is $\Delta p / p \approx 3 \%$. A detailed description of the detector ensemble can be found in [13]. In 2007 a measurement of proton-proton collisions at a kinetic beam energy of 3.5 GeV was performed: a beam with an average intensity $\sim 1 \times 10^{7}$ particles/s was incident on a liquid hydrogen target with a density of $0.35 \mathrm{~g} / \mathrm{cm}^{2}$ and a total interaction probability of $\sim 0.7 \%$. In total, $1.2 \times 10^{9}$ events were collected. The first-level trigger required at least three hits in the Time-of-Flight wall.

\section{Reconstruction of the $\Lambda(1405)$ line shape}

The reaction $p+p \rightarrow p+\Lambda(1405)+K^{+}$was analysed $[8,9]$ and the resulting missing mass spectrum $M M\left(p, K^{+}\right)$, showing a signal of the $\Lambda(1405)$, is presented on Fig. 1 . The most interesting finding is the location of the $\Lambda(1405)$ mass peak — at low mass of around $1385 \mathrm{MeV} / c^{2}$. This is the first reconstruction of the $\Lambda(1405)$ in proton-proton collisions in the charged decay mode and these data impose new constraints for models describing the production of $\Lambda(1405)$ in protonproton collisions.

A possible interpretation of the observed low-mass $\Lambda(1405)$ peak has been proposed in [10] as an effect of the interference of the $\Lambda(1405)$ with the non-resonant $\Sigma^{ \pm} \pi^{\mp}$ background. Here, the $\Lambda(1405)$ was modelled as a coherent sum of two Breit-Wigner functions, corresponding to 


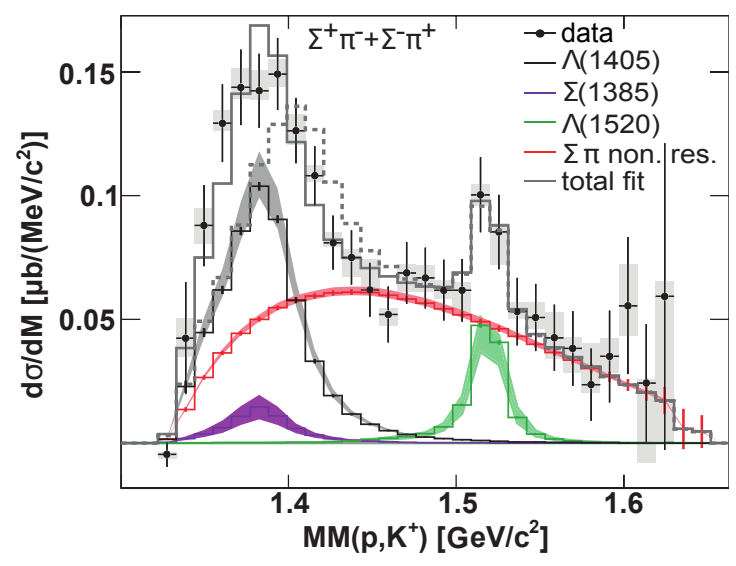

Figure 1: Sum of missing mass $M M\left(p, K^{+}\right)$distributions reconstructed with the $\Sigma^{+} \pi^{-}$and $\Sigma^{-} \pi^{+}$pairs [9]. The gray band shows the contribution of the $\Lambda(1405)$.

the two poles, positions of which were constrained by the recent calculations done in [11]. The non-resonant $\Sigma^{ \pm} \pi^{\mp}$ background was modelled as a 4th order polynomial. These two contributions $(\Lambda(1405)+$ background) were added up coherently with a complex mass-independent relative phase (a "maximum interference" scenario), which was set free as a fit parameter. Additional contributions of the $\Sigma(1385)^{0}$ and $\Lambda(1520)$ were added up incoherently. The result of the successful fit is shown in Fig. 2. Although a number of strong assumptions has been made in this simplified consideration, it shows that the observed low mass of the $\Lambda(1405)$ peak might be caused by an interference of the initial $\Lambda(1405)$ amplitude that exhibits a maximum at $1400 \mathrm{MeV} / c^{2}$ with the non-resonant background. Other scenarios are also considered in [10], including the possibility that the spectral shape of the $\Lambda(1405)$ in $p+p$ reactions gets more influenced by the presence of the broad $\Sigma \pi$ peak.

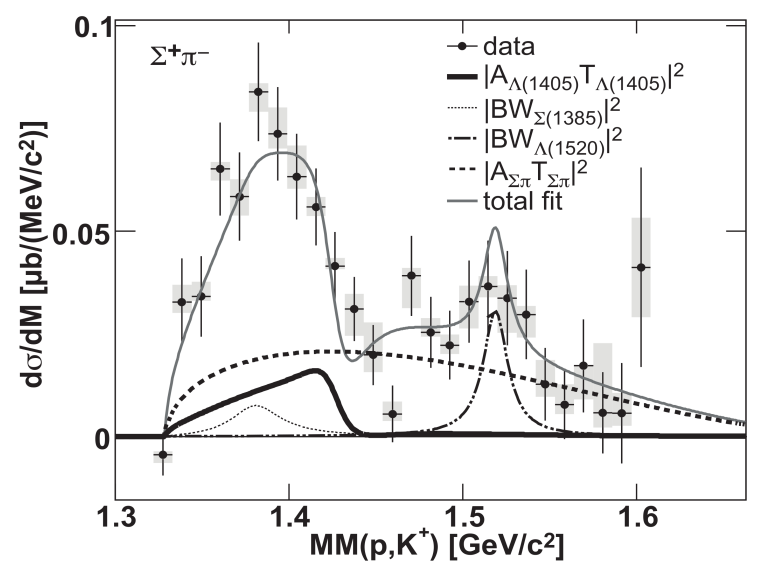

Figure 2: Missing mass spectrum to proton and $K^{+}$. The black data points are the measurements of [9], for the $\Lambda(1405)$ in the $\Sigma^{+} \pi^{-}$decay channel. The thin solid curve corresponds to the result of the fit within the scenario of a maximum interference of the $\Lambda(1405)$ amplitude (thick solid curve) and the non-resonant $\Sigma^{ \pm} \pi^{\mp}$ background (dashed curve). 


\section{Search for the $p p K^{-}$state}

In case the antikaon-nucleon attraction is strong enough, a system of two nucleons bound by an antikaon, a so-called $p p K^{-}$state might exist, as has been proposed in $[3,4,5]$. Most contemporary theoretical works (cf. [12] and references therein) predict that such a system is indeed bound. The ranges of predicted binding energies and widths are, however, very broad: $B \in[16,80] \mathrm{MeV} / c^{2}$ and $\Gamma \in[35,110] \mathrm{MeV} / \mathrm{c}^{2}$.

The expected decay channel $p p K^{-} \rightarrow p+\Lambda$ motivates the search for this state in the reaction $p+p \rightarrow p+K^{+}+\Lambda$. A dedicated analysis allowed to reconstruct $\sim 20 \times 10^{3}$ exclusive $p K^{+} \Lambda$ reactions with - owing to a good momentum resolution of the detector - a high purity.

In order to search for the $p p K^{-}$signal as a peak in the invariant mass distribution of the proton$\Lambda$ pairs a good understanding of the background sources is mandatory. A complication due to the role of $N^{*}$ resonances $\left(N^{*}(1650), N^{*}(1720), \ldots\right)$ arises here. Indeed, the decay mode $N^{*} \rightarrow \Lambda K$, is a major source of open strangeness production in the considered energy range. Contributing $N^{*}$ resonances will not only appear as broad structures in the invariant mass spectrum of decay products (i. e. $\Lambda$ - $K$ pairs), but also, as kinematic reflections, in the proton- $\Lambda$ invariant mass spectrum, hampering the extraction of the $p p K^{-}$signal, or, potentially, producing a fake signal. Therefore, a firm control over the background shaped by decays of $N^{*}$ resonances is a key issue in this search.

In order to take into account the contribution of the $N^{*}$ resonances (including interference effects) a Partial Wave analysis (PWA) of the reaction $p+p \rightarrow p+K^{+}+\Lambda$ has been performed using the Bonn-Gatchina PWA framework [14]. A set of resonances plus non-resonant waves was included in the solution. In fact, it was found that no unique solution can be obtained. Instead, a set of best solutions that differ in their resonance content has been chosen. First, a null-hypothesis has been considered and no contribution from the $p p K^{-}$state has been taken into account, but only conventional sources.

The resulting description of the $\mathrm{p}-\Lambda$ invariant mass spectrum is shown in Fig. 3. The experimental data are compared to the four best solutions (represented by the gray band) extracted from the PWA. Apparently, a good description of the data within the null-hypothesis is achieved, leaving little room for the $p p K^{-}$signal. A corresponding upper limit for the kaonic cluster formation is currently under evaluation.

\section{Summary}

The HADES collaboration reconstructed for the first time the contribution of the $\Lambda(1405)$ in the spectrum of $\Sigma^{ \pm} \pi^{\mp}$ pairs produced in proton-proton collisions. The observed $\Lambda$ (1405) mass peak is located around $1385 \mathrm{MeV} / \mathrm{c}^{2}$. The interference with the non-resonant background might play an important role in the generation of the observed line shape and must be considered by theoretical calculations.

The study of the sub-threshold $\bar{K} N$ interaction is continued with a Partial Wave analysis of the reaction $p+p \rightarrow p+K^{+}+\Lambda$ with the aim to find a footprint of the kaonic bound state formation. No clear structure associated with the $p p K^{-}$has been observed and an upper limit for the $p p K^{-}$ production will be reported in a dedicated publication. 


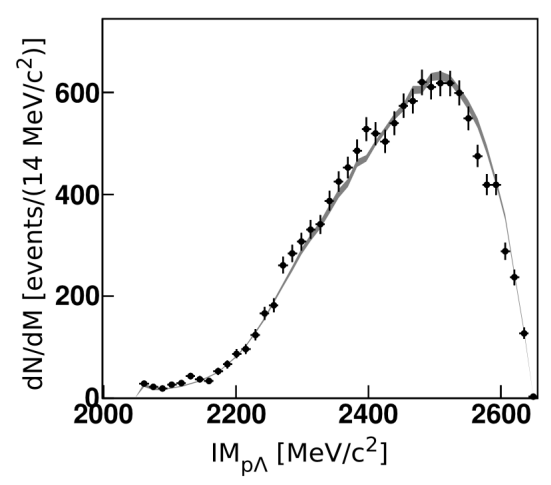

Figure 3: Invariant mass distribution of proton- $\Lambda$ pairs in the reaction $p+p \rightarrow p+K^{+}+\Lambda$. Symbols show experimental data. The gray band corresponds to a set of four PWA solutions obtained without inclusion of the hypothetical $p p K^{-}$state.

\section{Acknowledgments}

The HADES collaboration gratefully acknowledges the support by the grants LIP Coimbra, Coimbra (Portugal) PTDC/FIS/113339/2009, SIP JUC Cracow, Cracow6 (Poland): N N202 286038 28-JAN-2010 NN202198639 01-OCT-2010, HZ Dresden-Rossendorf (HZDR), Dresden (Germany) BMBF 06DR9059D, TU München, Garching (Germany) MLL München: DFG EClust 153, VH-NG-330 BMBF 06MT9156 TP5 GSI TMKrue 1012 NPI AS CR, Rez, Rez (Czech Republic) MSMT LC07050 GAASCR IAA100480803, USC - S. de Compostela, Santiago de Compostela (Spain) CPAN:CSD2007-00042, Goethe University, Frankfurt (Germany): HA216/EMMI HIC for FAIR (LOEWE) BMBF:06FY9100I GSI F\&E.

\section{References}

[1] B. Borasoy, U. -G. Meissner and R. Nissler, Phys. Rev. C 74, 055201 (2006) [hep-ph/0606108].

[2] T. Hyodo and W. Weise, Phys. Rev. C 77, 035204 (2008) [arXiv:0712.1613 [nucl-th]].

[3] Y. Nogami, Phys. Lett. 7, no. 4, 288 (1963).

[4] Y. Akaishi and T. Yamazaki, Phys. Rev. C 65, 044005 (2002).

[5] T. Yamazaki and Y. Akaishi, Phys. Lett. B 535, 70 (2002).

[6] G. Agakishiev et al. [HADES Collaboration], Phys. Rev. C 85, 035203 (2012).

[7] E. Epple and L. Fabbietti for the HADES Collaboration, Hyperfine Interact. 210, 45 (2012).

[8] G. Agakishiev et al. [HADES Collaboration], Nucl. Phys. A 881, 178 (2012).

[9] G. Agakishiev et al. [HADES Collaboration], Phys. Rev. C 87, 025201 (2013).

[10] J. Siebenson and L. Fabbietti, Phys. Rev. C 88, 055201 (2013).

[11] Y. Ikeda, T. Hyodo and W. Weise, Nucl. Phys. A 881, 98 (2012) [arXiv:1201.6549 [nucl-th]].

[12] A. Gal, Nucl. Phys. A 914, 270 (2013) [arXiv:1301.2145 [nucl-th]].

[13] G. Agakishiev et al. [HADES Collaboration], Eur. Phys. J. A 41, 243 (2009) [arXiv:0902.3478 [nucl-ex]].

[14] A. V. Anisovich, V. V. Anisovich, E. Klempt, V. A. Nikonov and A. V. Sarantsev, Eur. Phys. J. A 34, 129 (2007). 